\title{
Editorial
}

\section{Algunas aproximaciones a una perspectiva intercultural: entre discursos generales de la educación y específicos centrados en la naturaleza de lo que se quiere enseñar}

\author{
Adela Molina-Andrade
}

Diferentes perspectivas alimentan las conceptualizaciones o aproximaciones sobre la interculturalidad, las cuales directamente inciden en la forma como es asumida; tales perspectivas y conceptualizaciones se constituyen también en un contexto general y específico para abordar la temática del Dossier del presente número de la revista. En este caso, interesan las Perspectivas y desafíos de la interculturalidad en la educación de las ciencias experimentales, la matemática y la tecnología.

En esta presentación se abordan diferentes planos de la problemática, por demás compleja, dada la importante producción general y específica de disciplinas que reflexionan directamente sobre la educación, la pedagogía y la didáctica, o que de manera indirecta afectan este importante campo académico de investigación. Es así que no se propone un texto a profundidad, ni tampoco de manera exhaustiva; solo se proponen varios énfasis para su abordaje, los cuales, se originan en los desarrollos de la Línea de Investigación: Enseñanza de las ciencias, contexto y diversidad cultural, en la cual participan múltiples e importantes actores. Así, se sugieren reflexiones críticas y desafíos en torno a varias perspectivas de un enfoque intercultural: contexto sociocultural de los países e implicaciones; la cuestión del "otro", lo propio y lo ajeno; perspectivas plurales institucionales y políticas públicas; y relaciones entre culturas y entre conocimientos.

\section{Enfoque intercultural en el contexto sociocultural de los países e implicaciones}

En este punto se quiere mostrar, que no es posible hablar de un enfoque intercultural, en particular en la educación. Para que este tenga alguna trascendencia importante y positiva en las vidas de las comunidades y las sociedades, es necesario considerar y tomar en cuenta las dinámicas socioculturales propias de cada una de ellas. Al respecto, Cabo y Enrique (2004), enuncian la necesidad de investigaciones 
enfocadas en las condiciones específicas de diversidad cultural de una sociedad dada, para determinar el tipo de educación que se debe ofrecer. Aspecto importante a considerar, ya que se da gran importancia a "objetivos educativos", que tienden a impedir el reconocimiento de los efectos de los factores socioculturales en la enseñanza y el aprendizaje (Molina, et al., 2014).

Tomando como referencia el origen de las poblaciones y la educación que se imparte en ellas, y de acuerdo con enfoques socioculturales, es posible decir que los sistemas educativos inciden en la transformación o no de las sociedades de las cuales forman parte, y que estos no son ajenos a las características concretas de las mismas. Valladares (2010) profundizando en este punto dice: "Lo anterior es así porque los seres humanos vivimos inmersos y formamos parte de una cultura, a partir de la cual organizamos nuestras vidas, relaciones y prácticas sociales en términos de un horizonte de sentido y significado que nos dota de una cierta identidad" (p. 1). Desde una perspectiva cognitiva, lo anterior también es importante y ha sido abordado por diferentes escuelas e investigadores. Este es el caso de Vygostky, quien a pesar de relacionar el funcionamiento de la mente con los contextos socio-históricos, no considera la complejidad de las diferencias culturales. La crítica de Wertsch permite comprender mejor la importancia de las relaciones, entre la naturaleza cultural de las sociedades y sus sistemas educativos. Wertsch (1996) anota que Vygotski "[...] tendía a ver las diferencias como si fuesen diferencias a lo largo de un único camino hacia un ideal universal [...]" (p. 184), pero en la actualidad varios investigadores socioculturales dirían que "[...] tales aproximaciones no son adecuadas para entender la complejidad de las diferencias culturales" (Molina et al., 2013).

Con respecto, a los mecanismos implicados en la interrelación entre sociedad, sistemas educativos y cultura, Molina, a partir de Forquin, anota que

[l]os significados, productos, valores, etc. adquiridos a lo largo del tiempo en una cultura y que en ella permanecen, considerados deseables pasan a ser parte del currículo. La selección expresa evaluación, validación y legitimación, en los cuales permanentemente están siendo emitidos juicios de valor (2000, p. 95).

Al respecto Williams (1958), citado por Forquin (1993) afirma que los contenidos de enseñanza constituyen el producto de una selección efectuada en el seno de una cultura y los factores culturales son los que gobiernan dicha selección. De acuerdo con lo anterior, a partir de la selección de contenidos efectuada se puede caracterizar toda escuela.

Continuando con Molina (2000) encontramos:

Dado que toda selección de contenidos educativos implica también toma de decisiones, dicha escogencia no puede ser entendida de manera unidireccional, así como tampoco la dirección realizada hacia los profesores y los estudiantes. Para Forquin (1993), la selección de contenidos al interior de una cultura posee una doble importancia, a través de ella se realiza una reelaboración de contenidos, los 
cuales son transmitidos a las nuevas generaciones. Pero la cultura no es transmitida como un repertorio simbólico unitario, tal transmisión está sujeta a los azares de la relación simbólica y a los conflictos de la interpretación. (p. 95).

En el caso de la educación en ciencias y la matemática, esta especificidad (cultura y sistemas educativos) es analizada por varios investigadores, que ponen como telón de fondo los contextos culturales y sus influencias en las visiones de mundo, las creencias, valores, cogniciones, entre otras; aspectos que deberían determinar orientaciones para la enseñanza. Wilson (1981), al respecto considera que "para que la enseñanza de las ciencias sea efectiva, se debe tomar en cuenta explícitamente el contexto cultural de la sociedad de quienes la necesitan, el cual, proporciona los escenarios para que dicha enseñanza sea útil [...]". Lewin (1990, citado por Wilson, 1981) anotó que todavía "muchos niños que estudian ciencias en los países en vías de desarrollo, según las evidencias anteriores, sugieren que la gran mayoría no domina más de una pequeña proporción de las metas propuesta para ellos". Cobern y Loving (2001) argumentan que "una visión de ciencia múltiple, servirá más a necesidades de estudiantes que vienen de entornos culturales diversos y ayudará a cambiar el efecto, culturalmente corrosivo, que la ciencia Occidental ha tenido sobre culturas no occidentales". Otra consideración, para tener en cuenta, se refiere a que los fracasos registrados cuando se transfieren los mismos planes de estudio a diversos contextos culturales, hacen suponer, que las concepciones de conocimiento y de naturaleza constituidas en la cultura de cada uno los países, a los cuales se trasladan tales currículos, pueden entrar en contradicción con aquellas concepciones conformadas en la cultura de base del país o países, en los cuales se origina el currículo transpuesto.

De manera más específica, Osuna (2013) analiza el contexto en el cual se utiliza el enfoque de la interculturalidad, desde la perspectiva de la naturaleza cultural de las sociedades en las cuales se despliega, retomando el contexto latinoamericano y español. En el caso de la educación intercultural en América Latina, muestra que su surgimiento está relacionado con los pueblos indígenas, que buscan revalorizar y conservar sus lenguas y culturas, aspectos que se redefinen en el contexto de las relaciones de los pueblos con el estado; con esta perspectiva ellos enfrentan visiones unificadoras de las sociedades nacionales; luchas que han sido apoyadas por organismos internacionales. Al respecto, Valladares (2014), analiza el enfoque intercultural considerando aspectos relacionados con lo político y ciudadano para el contexto Latinoamericano, al entender que una institución educativa auténticamente intercultural y bilingüe debe garantizar que toda persona tenga libertad de escoger su identidad cultural y desarrollarla, sin sentirse discriminada y/o excluida. Aunque exista una amplia discusión sobre la refundación en estados plurales en Latinoamérica, en realidad la crítica sobre su naturaleza homogénea y opresora lo impide (Villoro, 1998).

También existe una importante producción en el campo de la etnomatemática y la enseñanza de las ciencias que aborda el enfoque intercultural, para señalar 
la importancia de considerar las perspectivas de los pueblos indígenas y afrodescendientes en diferentes lugares del mundo. Una de las primeras referencias (pero no la única) es la de Maddock (1981) quien, ante la poca información de tipo antropológico que permitiera orientar la formación científica a estudiantes de Padua Nueva Guinea, anota que:

Es fácil ser sabio con el beneficio de la retrospectiva, pero la entrada de los investigadores con investigaciones de naturaleza antropológica, implica que son conscientes del pasado, por tanto probablemente habría sido de mucho más valor, enfocarse en este aspecto, para el desarrollo del currículo de ciencias en los países del Tercer Mundo, que muchos de los esfuerzos de los expertos insensibles que tratan de forjar un currículo americano o británico. (p. 19).

Pero también, de otra parte, se identifican enfoques que buscan incorporar los conocimientos tradicionales en la enseñanza. Costa, Nascimento y Catarino, (2017), en su sinopsis sobre la incorporación de conocimientos etnomatemáticos en la enseñanza, establecen que existen dos aspectos implicados: el saber hacer y los saberes de los artesanos en el desempeño de sus labores, y la matemática escondida en los artefactos que ellos construyen en la realización de sus labores. El levantamiento de estos conocimientos y saberes permite la proyección de la enseñanza. Chinn (2012) da importancia a los conocimientos locales, y al aprendizaje situado (concordando con Costa, Nascimento, y Catarino, 2017); al respecto muestra diferentes planteamientos que fortalecen la relación entre conocimientos profesionales docentes y el lugar en donde la actividad docente se realiza. Propone incorporar, mediante el conocimiento de las condiciones locales de las comunidades los recursos de la cultura, las propias historias; lo cual aumentaría aprendizajes auténticos de los estudiantes indígenas y marginados. Cajete (1999) señaló que los indígenas americanos entendieron que una relación íntima entre ellos mismos y su entorno era la esencia de su supervivencia y su identidad como pueblo. Hizo hincapié en el potencial que poseen las prácticas, valores y conocimientos indígenas sobre el "lugar" que, a largo plazo, pueden informar a la ciencia occidental mediante investigaciones participativas orientadas hacia la sostenibilidad. Barnhardt y Kawagley (2005), muestran que los pueblos indígenas han tenido sus propias formas de mirar y relacionarse con el mundo, el universo; procesos tradicionales de una educación cuidadosamente construidos. Sin embargo, las visiones del mundo indígenas y sus propios enfoques educativos están en peligro, con la propagación de estructuras sociales occidentales y sus formas institucionalizadas de transmitir la cultura'.

En el caso de España, continúa Osuna (2013), la educación intercultural surge para dar respuesta a la aparición en las aulas de "poblaciones diferentes a la usual". Así, apoyada en varias referencias (Gil-Jaurena, 2008; Dietz y Mateos-Cortés, 2009), muestra que esencialmente la educación intercultural se 
centra en categorías como "inmigrante" y gitanos, que aunque expresan ideas "no categóricas" y abiertas sobre el significado de la diversidad cultural y la interculturalidad, no dan cuenta claramente de las "personas", de los grupos específicos o los grupos minoritarios. Benarroch (2001), anota que existen dos tipos de extranjeros en España: los europeos (gran mayoría) y los no europeos, provenientes de los países llamados del Tercer Mundo:

Los primeros no han suscitado estrategias interculturales que procuren su adaptación a la realidad española; los hijos de estos extranjeros han sido escolarizados en las escuelas españolas sin grandes problemas de adaptación y de integración, mientras que los procedentes del Tercer Mundo han originado un discurso "intercultural" intenso. (p. 12).

En este caso, el enfoque intercultural asociado a la diversidad cultural se entiende como demanda (enfoque diferencial) o como respuesta, que atiende a las poblaciones pertenecientes a otros grupos étnicos. Los trabajos de Benarroch (2001), Cabo y Enrique (2004) se basan en la caracterización de una ciencia intercultural.

\section{Enfoque intercultural y la cuestión del otro}

Las tensiones entre lo propio y lo ajeno en contexto (que implican relaciones con el otro y lo otro), configuran nuevas identidades y escenarios de acción, y es a lo que García (2004) se refiere cuando conceptualiza la interculturalidad como patrimonio. Así, conocer al otro, es tratar con su diferencia "se trata de enfrentar las nuevas complicaciones de la diversidad, evitando una búsqueda de la naturaleza humana descontextualizada" (p. 55). Así, "la interculturalidad remite a la confrontación y entrelazamiento, a lo que sucede cuando los grupos entran en interacciones e intercambios. Es así como la [...] interculturalidad implica que los diferentes son lo que son en relaciones de negociación, conflictos y préstamos recíprocos" (García, 2004, p. 15).

De acuerdo con Díaz-Crovetto y Samaniego (2015), se requiere una reflexión crítica sobre el uso del término intercultural, la cual implica que los procesos que se producen deben constituirse en efectivos y "que busquen generar transformaciones socioculturales al servicio de una mayor justicia distributiva y cultural (Frazer, 2008) contemplando [...] las reflexiones desde diferentes disciplinas sobre la relación de lo propio y lo extranjero; y de otra parte, sobre cómo los procesos, acciones de distinta naturaleza logran constituirse en caja de resonancia de las dinámicas que se dan en el mundo de lo cotidiano" (Díaz-Crovetto y Samaniego, 2015 , p. 88). Al respecto, estos mismos autores aducen que:

uno de los epistemes originales de la antropología trató de la construcción

teórica y metodológica para entender al otro a partir de un contacto cultural que reafirmó la diversidad y la pluralidad de otros, y con el tiempo de nosotros. 
cuestionamientos, diferenciándose entre estudios sobre el otro, con el otro, para

el otro o desde el otro. (Díaz-Crovetto y Samaniego, 2015; p. 94).

La reflexión sobre el "otro" permite un acercamiento al tema de la subjetividad, la identidad cultural y la concepción de un sujeto localizado y contextualizado en un marco de interpretación cultural (Molina, 2005, p. 139). Dicha reflexión sobre el otro, ha motivado puntos de vista y argumentos cuando se analiza la enseñanza implementada en sociedades, pueblos y comunidades no occidentales u "occidentalizadas", que señalan la necesidad de una comprensión crítica sobre la enseñanza, los cuales involucran aspectos ideológicos y políticos (Molina y Mojica, 2013). Al respecto, Riggs (2005), Medina-Jerez (2008) y Mckinley (2008) consideran que, al no identificarse tales aspectos ideológicos y políticos en las propuestas educativas, terminan promoviendo modelos coloniales de enseñanza. Es indudable la necesidad de incluir, además de los conocimientos científicos escolares, variados sistemas de conocimiento como los indígenas u otros no occidentales.

[...] en tal sentido, Hodson (1999); Kawagley, Norril-tull y Norris-tull (1998); Snively y Corsiglia (2001); Stanley y Brickhouse (2001) están motivados por un deseo de alterar el panorama político de la educación científica. Interesa tenerlo presente en los análisis sobre cómo el ejercicio del poder permite o propicia relecturas de la globalización ("modernidad", "relación centro-periferia", "cerrar la brecha", "relación local-universal"). (Molina et al., 2013, p. 42).

Aikenhead y Ogawa (2007) agregan que la exclusión de los conocimientos nativos se ha naturalizado, mediante la colonización que ha ejercido la ciencia occidental moderna, refiriéndose a los pueblos nativos de Alaska, al considerar que la Ciencia se trata de conocimientos Universales, cuya caracterización no considera los contextos histórico-políticos, a diferencia de los conocimientos nativos que son vinculados de manera específica a los mismos. Lo anterior le confiere una superioridad a los primeros, que interioriza a los segundos; se trata de una historia de silenciamiento de otros conocimientos basada en una comprensión inadecuada de tales contextos. Además de la comprensión del otro, sin la explicitación de aspectos ideológicos y políticos de discursos que naturalizan la exclusión.

También esta perspectiva tiene repercusiones en el enfoque intercultural. Díaz-Crovetto y Samaniego (2015) llaman la atención sobre el inusitado uso de la interculturalidad en el campo académico, las políticas públicas internacionales, las ONG, las perspectivas neoliberales, su uso descontextualizado, el cual no puede entenderse como un término neutro que se utiliza desconectado de cualquier dimensión ética; basado en Onghena (2014), entiende la interculturalidad como la existencia y la necesidad de la misma concebida como vivencia de la interdependencia con el otro y conciencia de esta vivencia en tanto condición de nuestra época. Así dice "De este modo, afirmaremos su existencia como algo en ocasiones no problemático, en otras buscado, en ciertas situaciones imposible, 
pero necesario, pero nunca deseable como ejercicio que en principio se proyecte desde un plano normativo" (p. 96).

Desde el punto de vista de la enseñanza y el aprendizaje de conocimientos, dos ideas son importantes para comprender la manera como se considera al otro, cuando entran en contacto conocimientos de diferentes culturas. Hills (1989, citado por Molina, s.f.) llama la atención sobre cómo los profesores tienden a enfocarse solamente en la explicación de conceptos científicos a "sujetos occidentales". Considera necesario adoptar el "enfoque de "conceptos extranjeros" que está basado en dos premisas: primero que la ciencia se realiza en un contexto cultural específico y, segundo que su enseñanza y su aprendizaje es a menudo una actividad de intercambio cultural, ya sea al interior de una cultura o entre culturas diferentes" (Molina, s.f.,). En cuanto al aprendizaje, Cobern (1994), basado en la idea según la cual el conocimiento y la creencia se puedan distinguir desde el punto de vista filosófico, tal diferenciación no es relevante en términos pedagógicos, ya que todas las personas tienen creencias fundamentales (no racionales/intuitivas) desde las cuales juzgan el conocimiento; es decir, de alguna manera, el conocimiento del sujeto descansa sobre la creencia. Así, propone una nueva forma de entender los aprendizajes, ya no se entenderían como la adopción de las creencias científicas, sino como la comprensión de las mismas, lo cual implica que no necesariamente debemos estar de acuerdo con ellas para aprenderlas; esta perspectiva da fuerza a la posibilidad de interactuar conjuntamente, sin el imperativo de olvidar o hacer a un lado las propias visiones de mundo para aprender otras.

\section{Perspectivas plurales, políticas públicas e interculturalidad}

Desde esta triple relación (pluralidad cultural, políticas públicas e interculturalidad), se propone buscar el respeto y el reconocimiento del otro. Se requieren diferentes esfuerzos para aproximarse a una educación que no discrimine otros conocimientos derivados de otras culturas y perspectivas sobre la naturaleza, que implica el replanteamiento de las prácticas educativas en general, y en particular de la enseñanza de las ciencias, las matemáticas y la tecnología (Valladares, 2014; Aikenhead y Huntley, 1999; Quintriqueo y Mcginity, 2009). Siguiendo a Benavides (2012) podemos decir que se requieren perspectivas pluralistas y participativas en las instituciones educativas, tanto en sus formas de organización, como en el orden curricular y en el ambiente institucional, para garantizar el reconocimiento y protección de la diversidad y diferencia étnica y cultural. Santiago, Akkari e Marques (2013) anotan que "la interculturalidad prospera en sociedades que se tornan pluralistas y democráticas" (p. 9).

Una comprensión del trasfondo de políticas públicas no plurales es aclarada por Valladares (2014), quien propone que la naturaleza homogeneizadora de los estados en América Latina se basa en la exclusión cultural, que surge de la falta de reconocimiento y respeto de las culturas de los otros. Así, el papel de educación intercultural y bilingüe se constituye en una de las vías para lograr 
estados plurales. Se considera que sería incompleto un proyecto académico y político de este tipo que no considere la enseñanza de las ciencias, la matemática y la tecnología para lograr la expansión de la libertad cultural de las personas, al respecto considera:

Así, para que la educación contribuya a la expansión de las libertades que una persona tiene realmente para hacer o ser lo que le resulta valioso, es preciso lograr la conversión de la pluralidad de recursos cognitivos que ofrecería una auténtica educación intercultural y bilingüe (y esto incluye a los conocimientos científicos y tecnológicos, pero también a los conocimientos locales, tradicionales, indígenas, entre otros), en oportunidades reales de vivir y de alcanzar aquello que se valora. Valladares $(2014$, p. 10).

Retomando la importancia de desarrollar todas las potencialidades humanas, Benarroch (2001) anota

el desarrollo de las potencialidades de todo el alumnado requiere la capacidad de diseñar un currículo capaz de transmitir mensajes antirracistas, que preferimos denominar interculturales o transculturales (estos términos, recogiendo las mismas incidencias educativas, conllevan menos prejuicios que en algún caso pueden ofender), capaces de romper con la hegemonía cultural [...]. (p. 14).

Como muchas perspectivas, investigaciones y acciones escolares y educativas, la interculturalidad es un discurso que busca la eliminación de la exclusión, al respecto en la literatura de la educación científica, tecnológica y matemática también se registra dicha tendencia. Benarroch (2001), quien está de acuerdo con Atwater y Riley (1993), anota que con la acepción intercultural se alude, generalmente, a grupos culturales como los inmigrantes y las mujeres, que generalmente presentan menores rendimientos en estas disciplinas y con una presencia también menor en el trabajo científico y tecnológico. Al respecto, Pringle y McLaughlin (2014) relacionan estas problemáticas con las ideas predominantes sobre las características de un científico, mostrando que en las aulas se pone en evidencia la nula participación de los grupos llamados como no dominantes y por el poco reconocimiento dado a la diversidad cultural. Históricamente, la ciencia ha sido vista por muchos como una cultura libre, de etnias y de género (Carter, Larke, Singleton-Taylor y Santos, 2003). En esta perspectiva difícilmente aparece en el imaginario de los profesores, la referencia a científicos afroamericanos, latinos, indígenas, asiáticos o mujeres.

Afortunadamente, un número creciente de investigadores de la educación en ciencias, matemáticas y tecnología reconocen que es necesario un cambio significativo en la forma que tradicionalmente estas disciplinas se han enseñado; transformando métodos de enseñanza que hacen hincapié en el blanco, macho, occidental, y las perspectivas europeas (Atwater, 1994, 1995; Carter, Larke, Singleton-Taylor y Santos, 2003). Por ejemplo, un componente clave en este cambio de paradigma es la necesidad de hacer hincapié en la pedagogía culturalmente 
relevante en el aula, una "pedagogía que se caracteriza por el empoderamiento individual y colectivo" (Ladson-Billings, 1995a, 1995b, 2000).

\section{Relaciones entre culturas y relaciones entre conocimientos}

Varias perspectivas y enfoques se han propuesto, las cuales permiten observar de manera crítica tales relaciones. Con Benarroch (2001), encontramos cuatro tipos de relaciones basadas en aspectos afectivos: (a) Asimilación, en la cual existe una tendencia a abandonar la identidad cultural de partida y la tendencia a aceptar la sociedad de acogida. Supone una pérdida de los valores culturales propios; (b) Integración que resulta de la dinámica de mantener la propia identidad cultural de partida e integrarse a la identidad cultural de la sociedad de acogida; (c) Desintegración (marginalización y exclusión) caracterizada por un bajo nivel de identidad étnica y de aculturación; hay un alejamiento de la propia cultura, como también un rechazo de la cultura del grupo mayoritario de la sociedad de acogida, y (d) Etnocentrismo (separación y segregación) se presenta cuando no hay relación con la sociedad de acogida y la propia, se mantiene la identidad y tradiciones propias. Es normal para una persona que lleva poco tiempo en la cultura de acogida, pero se impone la identidad de la sociedad de acogida.

Molina y Mojica (2013) basan su caracterización de las relaciones interculturales en cuatro procesos de enseñanza, que lo entienden como puentes: (a) En la perspectiva asimilacionista, el conocimiento científico es el punto de partida y de llegada de la enseñanza de las ciencias. En esta perspectiva, el interés es reducir, asimilar o no considerar los conocimientos ecológicos tradicionales; (b) La perspectiva moral y humanista, se fundamenta en el reconocimiento del otro, circunscrito a lo moral y humanista; sin embargo, esta aproximación al otro no considera las relaciones entre conocimientos; (c) En la perspectiva plural epistemológica y ontológica, los puentes se configuran con la pretensión de argumentar e implementar los intercambios entre los conocimientos ecológicos y los científicos escolares, involucrando la alteridad relacionada con aspectos epistemológicos y ontológicos. Esta perspectiva implica dos aspectos, por una parte, la manera de entender y tratar las experiencias, saberes, conocimientos ancestrales y tradicionales y, por otra, casos particulares de interacciones de conocimientos y perspectivas distintas en la clase; (d) Perspectiva contextual, en esta el contexto puede configurarse como el puente mismo, se refiere a la configuración de un contexto para la emergencia de diferentes conocimientos y experiencias.

Finalmente, El-Hani y Sepúlveda (2006); Molina et al. (2009), sintetizan los debates epistemológicos en torno a enseñanza en cuatro tendencias: (a) Universalista, sostiene que la realidad es árbitro final de las adecuaciones de los enunciados, lo cual le confiere a la ciencia, la matemática y la tecnología un poder epistémico superior en relación con otras formas de conocimiento; (b) Multiculturalista, la cual se refiere a que el universalismo y la política de exclusión, que este implica, es incorrecta desde el punto de vista epistemológico, moral y político, y proponen 
la inclusión de los conocimientos tradicionales en el currículo; (c) Pluralista epistemológica, sostiene que todas las ideas no son iguales, que no todas las formas de pensar son paralelas y se compromete con la participación y compromiso civil, con base en nuestras diferencias y desacuerdos sobre lo que entendemos como verdad. La intención es fomentar este pluralismo que siempre nos permite revivir y sostener la conversación acerca de lo que realmente importa sobre lo que se considera, es la verdad; y (d) Interculturalistas, se basan en que el cruce de fronteras culturales es un fenómeno que ocurre continuamente en cualquier aula de clase, inclusive en sociedades fundamentalmente urbanas; reconocen que tales cruces deben ser estudiados e incorporados a la clase, como una potencialidad en el proceso de enseñanza y aprendizaje.

Dossier. El contexto presentado en torno a los cuatro aspectos tratados se basa en cuatro trabajos:

- Saber ancestral y conocimiento científico entre tensiones interculturales e identidades: una experiencia educativa para el caso del oro en Colombia en estudiantes de educación media, de Yolanda Dueñas Porras y Andrea Aristizábal Fúquene. Aborda el poco reconocimiento y visibilización de los saberes producidos en nuestras regiones. Se trata de una investigación cualitativa, con un enfoque socio-crítico; se refiere a un trabajo de aula con estudiantes de una institución educativa de décimo grado. Se articula una perspectiva intercultural y se retoman aspectos de la sociología de la ciencia, la ecología de saberes, las epistemologías del sur, la didáctica del patrimonio y la didáctica de las ciencias. Con respecto a los hallazgos, las autoras reportan dos tipos de tensiones interculturales: Acontecimiento social y cultura occidental, saber ancestral y conocimiento científico. Aspectos que se vinculan con variados niveles de identidad patrimonial como: ausente, latente patente y extendida (basados en la propuesta de Boaventura de Sousa en Epistemologías del Sur). De igual manera, consideran como otro hallazgo la importancia de aproximar más la ciencia a la realidad de los estudiantes, con lo cual se contribuye al rescate del saber ancestral y su historia; el estudiante se constituye en cohesionador de la ciencia, su cultura y la identidad patrimonial. Así, se generan aportes a la didáctica de las ciencias.

- Los puentes en la enseñanza de las ciencias: un compromiso para comprender las investigaciones sobre las relaciones entre conocimientos científicos escolares y conocimientos ecológicos tradicionales de Nadenka Beatriz Melo Brito. Parte de una realidad, ante la cual la enseñanza de las ciencias no puede ser ajena, como es la diversidad cultural en nuestro país (Colombia). Se propone entonces, como alternativa para garantizar la inclusión de los conocimientos tradicionales en diálogo con los conocimientos científicos escolares, la categoría de puentes; y dicho artículo significa un avance en su conceptualización. En tal sentido, su revisión se realiza a partir de la pregunta: ¿̇Qué orientaciones encontramos en los 
trabajos de investigación sobre puentes entre conocimientos científicos escolares y conocimientos ecológicos tradicionales en la enseñanza de las ciencias? Al respecto identifica dos perspectivas: (a) cómo metáfora (importante para la comprensión) que permite la elaboración de significados que se manifiestan en el aula, acercan la vida cotidiana y la clase de ciencias; y (b) como representación que intervienen en la elaboración de conceptos en la enseñanza de las ciencias, poniendo en contacto las culturas y contextos de quién aprende, sus visiones de mundo, entre otras.

- Affordance: Constructo para la comprensión y transformación de los procesos de enseñanza-aprendizaje y producción de saber en contextos interculturales interconectados, de Julio Ernesto Rojas Mesa y Linda Alejandra Leal Urueña. Se desarrollan dos líneas de reflexión: una relacionada con la producción de saber en contextos interculturales y asociada con los procesos de aprendizaje en la era digital. El ámbito de este trabajo se refiere a la educación basada en las conexiones e iterconexiones mediadas por los ambientes digitales y las TIC, y en una formación que reconozca la diversidad étnica y cultural. En este marco nos dicen los autores que el "concepto de affordance en el marco de la diversidad social y cultural potencia los procesos de producción de saber local, así como los intercambios de saberes que propician escenarios interculturales para la innovación de los procesos de enseñanza-aprendizaje y producción de saber". De otra parte, este concepto desde la prespectiva del diseño como innovación para poner en relación personas, artefactos y ambientes, supera los abordajes teóricos en tanto que existen escasos desarrollos en la práctica e innovación educativa.

- La historia oral en las memorias de prácticas culturales para pensar un (por)venir de la educación (matemática) indígena de Carolina Higuita, Abelardo Tascón, Diana Jaramillo. Este artículo se refiere a una investigación en el campo de la etnomatemática, y busca visibilizar las tensiones y desafíos que implica el hacer (y el ser) investigación, en este campo. Esta investigación se refiere a la movilización de objetos culturales desde la memoria de la práctica, en la construcción de una vivienda tradicional Embera-Chamí. La reflexión en torno a dichas tensiones se realiza desde las posibilidades que brinda la metodología de historia oral; así identificaron tres tensiones. Estas se refieren a: a) implicada en la constitución de la relación entre comunidad indígena e investigadores; (b) relacionada con la reconsideración de la lengua y los diferentes lenguajes que amplian los horizontes teóricos, metodológicos y prácticas de investigación etnomatemática, y (c) los nuevos aprendizajes y redefiniciones de otras formas de ser y estar en el mundo inspiradas en la sabiduría indígena ancestral. El método de Historia Oral desarrollado en la cultura occidental, en diálogo con las formas propias de hacer investicación de la comunidad, permitió la valoración de la experiencia desde las propias voces de los propios actores, voces usualmente sileciadas y oprimidas. 


\section{Referencias}

Atwater, M., y Riley, J. (1993). Multicultural Science Education: Perspectives, definitions and research agenda. Science Education, 77(6), 661-668.

Atwater, M. M. (1994). The multicultural science classroom part I: Meeting the needs of a diverse student population. The Science Teacher, 62(2), 20-23.

Atwater, M. M. (1995). The multicultural science classroom part II: Assisting all student with science acquisition. The Science Teacher, 62(4), 42-45.

Aikenhead, G., y Huntley, B. (1999). Teachers' views on aboriginal students learning western and aboriginal science. Canadian Journal of Native Education, 23(2), 159-175.

Aikenhead, G. S. y Ogawa, M. (2007). Indigenous Knowledge and Science Revisited. Cultural Studies of Science Education, 2, 539-620.

Barnhardt, R., y Kawagley, A. O. (2005). Indigenous knowledge systems and alaska native ways of knowing. Anthropology and Education Quarterly, 36(1), 8-23.

Benarroch, A. (2001). Interculturalidad y enseñanza de las ciencias. Alambique, 29, 9-23.

Benavides, E. (2012). La universidad pública y la diversidad étnica y cultural a la luz de la constitución política de Colombia de 1991. Estudios de Derecho, 59(154), 209-233.

Cabo, J., M. y Enrique, C. (2004). Hacia un concepto de ciencia intercultural. Enseñanza de las ciencias, 22(1), 137-146.

Cajete, G. (1999). Native Science. Natural Laws of Interdependence. New Mexico: Clear Light Publishers.

Carter, N. P., Larke, P. J., Singleton-Taylor, G., y Santos, E. (2003). Multicultural science education: Moving beyond tradition. In M. Hines (Ed.), Multicultural science education: Theory, practice, and promise (pp. 1-19). New York: Peter Lang.

Chinn, P. (2012). Developing teachers' place-based and culture-based Pedagogical Content Knowledge and agency. En B. Fraser, K. Tobin y K. McRobbie, Second International Handbook of Science Education. New York: Springer Dordrecht Heidelberg.

Cobern, W. (1991). World View Theory and Science Education Research. ManhattanKansas: NARST.

Cobern, W. (1994). Point: Belief, understanding, and the teaching of evolution. Journal of Research Science Teaching, 31, 583-590.

Cobern, W. \& Aikenhead, G. (1998). Cultural Aspects of Learning Science. En Fraser y Tobin (Eds.), International Handbook of Science Education. London: Kluwer Academic Publisher. 
Cobern, W., y Loving, C. (2001). Defining "science" in a multicultural world: Implications for science education. Science Education, 85, 50-67.

Costa, C., Nascimento, M., y Catarino, P. (2017). Sinopse dos estudos sobre (etno) saberes matemáticos efetuados no nordeste português e sua aplicação didática. Revista Latinoamericana de Etnomatemática, 10(1), 128-136.

Díaz-Crovetto, G., y Samaniego, G. (2015). El interminable apogeo de la interculturalidad: algunas reflexiones críticas desde la antropología y la filosofía. Tabula Rasa, 22, 85-102.

Dietz, G. y Mateos-Cortés, L. (2009). El discurso intercultural ante el paradigma de la diversidad. En T. Aguado Odina y M. del Olmo (Coords.), Educación intercultural. Perspectivas y propuestas (pp. 47-67). Madrid: UNED.

El-Hani, N. C., y Sepúlveda, C. (2006). Referenciais teóricos y subsídios metodológicos para a pesquisa sobre as relações entre educação científica e cultura. En F. Teixeira Dos Santos y M. A. Greca. Pesquisa em ensino de ciências no Brasil e suas metodologias (pp. 161-212). Rio Grande do Sul: Unijuí Editora.

Forquin, Jean-Claude. (1993). Escola e cultura. As bases sociais e epistemológicas do conhecimento escolar. Porto Alegre: Artes Médicas.

García Canclini, N. (2004). Diferentes, desiguales o desconectados. Revista CIDOB d'Afers Internacionals, 66-67, 113-133.

Gil-Jaurena, I. (2008). El enfoque intercultural en Educación Primaria: una mirada sobre la práctica escolar. Madrid: UNED.

Kawagley, A. O., Norril-Tull, D., y Norris-Tull, R. A. (1998). The indigenous worldteaching of science. Journal of Research in Science Teaching, 35(2), 133-144.

Ladson-Billings, G. (1995a). Toward a culturally relevant pedagogy. American Educational Research Journal, 32(3), 465-491.

Ladson-Billings, G. (1995b). But that's just good teaching! The case for culturally relevant pedagogy. Theory into Practice, 34(3), 159-165.

Ladson-Billings, L. (2000). Fighting for our lives: Preparing teachers to teach African American students. Journal of Teacher Education, 51 (3), 206-214.

Maddock, M. N. (1981). Science education: An anthropological viewpoint. Studies in Science Education, 8, 1-26.

McKinley, E. (2008). From object to subject: Hybrid identities of indigenous women in science. Cultural Studies in Science Education, 3(4), 959-975.

Medina-Jerez, W. (2008). Between local culture and school science: The case of provincial and urban students from eastern Colombia. Research in Science Education, 38(2), 189-212. 
Molina, A. (2000). Conhecimento, Cultura e Escola: Um estudo de suas Inter- relações a partir das idéias dos alunos (8-12 anos) sobre os espinhos dos cactos (tesis doctoral). Faculdade de Educação, Universidade de São Paulo, Brasil.

Molina, A. (2005). El "otro" en la constitución de identidades culturales. En Piedrahita, C. \& Paredes, E. (Eds.), Cultura política, identidades y nueva ciudadanía, vol. 2 (pp.139-169). Cúcuta: Sic Editorial.

Molina, A. (s.,f.). Línea de Investigación Enseñanza de la ciencias, contexto y diversidad cultural, énfasis Educación en ciencias, DIE-UD. Recuperado de http:// die.udistrital.edu.co/sites/default/files/doctorado_ud/lineas_inv/documentos/ ensenanza_ciencias_contexto_y_diversidad_cultural.pdf.

Molina, A., Martínez, C. A., Mosquera C. J. \& Mojica, L. (2009). Diversidad cultural e implicaciones en la enseñanza de las ciencias: reflexiones y avances. Revista Colombiana de Educación, 56, 103-128.

Molina, A., Pérez, R., Bustos, E., Castaño, C., Suárez, O., J. y Sánchez, M. (2013). Mapeamento informacional bibliográfico de enfoques e campos temáticos da diversidade cultural: o caso dos journal CSSE, Sci. Edu. e Sci \& Edu. Recuperado de http://www.nutes.ufri.br/abrapec/ixenpec/atas/resumos/R1641-1.pdf

Molina, A., y Mojica, L. (2013). Enseñanza como puente entre conocimientos científicos escolares y conocimientos ecológicos escolares. Magis, 6(12), 37-53.

Molina, A., Mosquera, C., J., Utges, G., R., Mojica, L., Cifuentes, M., C., Reyes, J., D., Martínez, C., A., y Pedreros, R., I. (2014). Concepciones de los profesores sobre el fenómeno de la diversidad cultural y sus implicaciones en la enseñanza de las ciencias. Editorial Universidad Distrital Francisco José de Caldas: Bogotá.

Onghena, Y. (2014). Pensar la mezcla. Un relato intercultural. Barcelona: Gedisa.

Osuna, C. (2013). En torno a la educación intercultural. Una revisión crítica. Revista de Educación, 358, 38-58.

Pringle, R. M., y McLaughlin, C. A. (2014). Preparing science teachers for diversity: Integrating the contributions of scientists from underrepresented groups in the middle school science curriculum. En M. Atwater, M. Russell, M. Butler (Eds.), Multicultural Science Education: Preparing teachers for equity and social justice (pp. 193-208). Dordrecht: Springer.

Quintriqueo, S., y Mcginity, M. (2009). Implicancias de un modelo curricular mono cultural en la construcción de la identidad sociocultural de alumnos/as mapuche de la IX región de la Araucanía, Chile. Estudios Pedagógicos, 35(2), 173-188.

Riggs, E. (2005). Field-based education and indigenous knowledge: essential components of geoscience education for native american communities. Science Education, 89(2), 296-313.

Santiago, C., M., Akkari, A., y Marques, P. L. (2013). Educação Intercultural. Petrópolis RJ: Editora Vozes. 
Valladares, L. (2010). Educación Intercultural Bilingüe: Una educación científica para la interculturalidad. Ponencia presentada en el Congreso Iberoamericano de Educación, Metas 2021. Buenos Aires República de Argentina, 13, 14 y 15 de septiembre de 2010. Recuperado de http://www.adeepra.org.ar/congresos/Congreso\%20 IBEROAMERICANO/INTERCULTURALBILINGUE/RLE2668_Valladares.pdf

Valladares, L. (2014). Educación y libertad cultural en México ¿̇Un camino abierto hacia los Estados plurales? Alteridad, 9(1), 8-18.

Villoro, L. (1998). Estado plural, pluralidad de culturas. México: Paidós/unam.

Wertsch, J. V. (1996). Un diálogo entre la Teoría sociocultural y la Psicología social actual. En. D. Paez y A. Blanco (Eds.), La teoría sociocultural y la psicología social actual (pp. 183-190). Madrid: Fundación Infancia y Aprendizaje.

Wilson, B. (1981). The cultural contexts of science and mathematics education: Preparation of a bibliographic guide. Studies in Science Education, 8, 27-44. 